CMEARTICLE

\title{
Clinics in diagnostic imaging (208)
}

Jin Rong $\underline{T a n}^{1}$, MBBS, FRCR, Adrian Cheng Kiang $\underline{L a u}^{2}$, MBBS, FRCSEd, Tien Jin $\underline{\operatorname{Tan}}{ }^{3}$, MBBS, FRCR
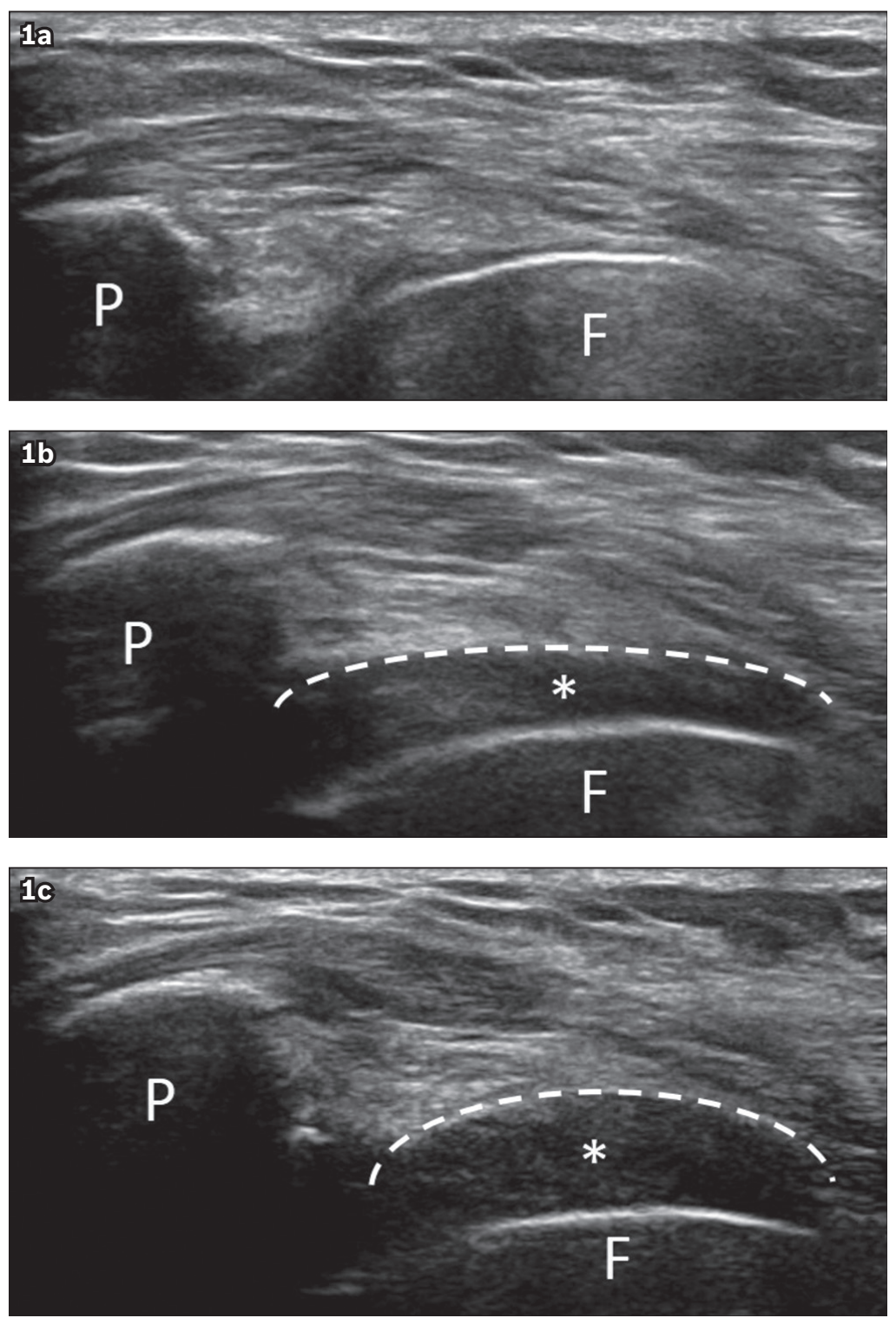

Fig. $1(\mathrm{a}-\mathrm{c})$ US images of the right knee. F: femoral component; P: patellar component.

\section{CASE PRESENTATION}

A 72-year-old woman who had total knee replacement (TKR) surgery two years prior presented with pain, weakness and an audible snap during extension of her right knee for the preceding 18 months. She was able to ambulate without aid, although climbing of stairs was only possible one step at a time due to her symptoms. There was no postoperative episode of infection or trauma affecting her right knee. Physical examination revealed a painful catch and audible snap above the patella during extension of the knee at about $30^{\circ}-40^{\circ}$. No obvious joint effusion was clinically evident. The patient underwent a greyscale ultrasonography (US) examination of her right knee. What do the US images in Fig. 1 show? 


\section{IMAGE INTERPRETATION}

The greyscale US images (Fig. 1), which were obtained with the right knee in (a) $90^{\circ}$ flexion, (b) $30^{\circ}-40^{\circ}$ extension and (c) full extension, demonstrate the sudden appearance of a focus of hypoechoic scar tissue (asterisk, outlined by white dashed line) in the suprapatellar region at $30^{\circ}-40^{\circ}$ of extension with perceivable 'snapping' of the knee by the patient, followed by further increased prominence of the scar tissue with the knee in full extension.

\section{DIAGNOSIS}

Right knee patellar clunk syndrome.

\section{CLINICAL COURSE}

The patient declined the option of surgical resection of the suprapatellar scar tissue, which was deemed to be the cause of her symptoms. Thus, she was managed conservatively and placed on a course of physiotherapy, leading to subsequent improvement of her symptoms.

\section{DISCUSSION}

Patellar clunk syndrome is the most common cause of perceivable 'snapping' of the knee following TKR. It is due to impingement of a suprapatellar nodule of scar tissue as it enters the prosthetic intercondylar notch with knee flexion, which then displaces with an audible and often painful 'clunk' with knee extension. ${ }^{(1)}$ It may be more prevalent in posterior-stabilised TKR, in which it has been reported in approximately $1 \%$ of cases and occurs within a few months to several years following surgery. ${ }^{(1,2)}$ Clinically, patients complain of painful catches or audible snaps in the suprapatellar region during extension of the knee at approximately $30^{\circ}-40^{\circ}$. Post-surgical risk factors that have been identified include a lowlying patella (patella baja) or proximal overhang of the patellar component of the TKR. ${ }^{(2)}$

Because of the significant imaging artefacts produced by the metallic TKR implants, ultrasonography is the preferred imaging modality of choice. Under real-time ultrasonography examination, a fibrous nodule is seen to suddenly displace from the intercondylar notch into the suprapatellar region with knee extension, while a snap is perceived under the probe and an audible 'clunk' may be perceptible. ${ }^{(3)}$ Magnetic resonance (MR) imaging may be useful in detecting the presence of the suprapatellar fibrous nodule if metallic artefact-reduction sequences are employed, although it has the disadvantage of a lack of dynamic assessment. ${ }^{(4)}$ The use of computed tomography $(\mathrm{CT})$ remains limited in the imaging assessment of patellar clunk syndrome. Open arthrotomy or arthroscopic excision of nodules is efficient for treating symptoms. ${ }^{(5)}$

Other commonly reported postoperative complications following TKR include polyethylene wear with or without osteolysis (25\%), aseptic loosening (24\%), instability and dislocation $(21 \%)$, prosthetic joint infection $(17 \%)$ and periprosthetic fracture $(3 \%) .{ }^{(6)}$

One of the most common causes of late TKR failure is aseptic loosening, which is postulated to result from cumulative mechanical stresses, osteolysis secondary to particle disease,
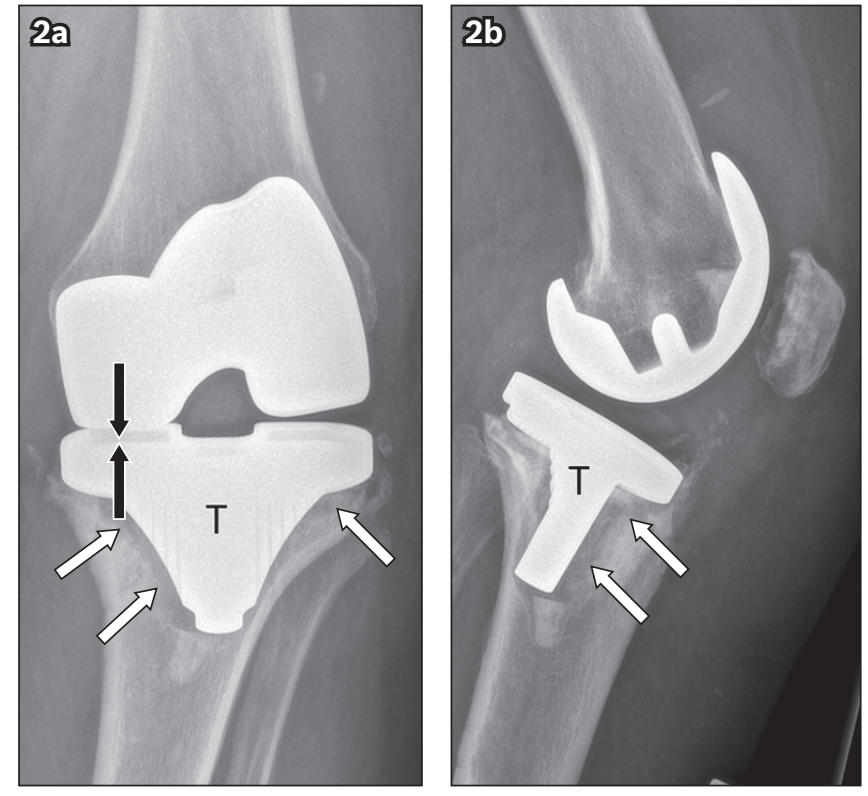

Fig. 2 (a) Anteroposterior and (b) lateral left knee radiographs in a 67-year-old woman with aseptic loosening show significant periprosthetic lucency (white arrows) surrounding the base plates and stem of the tibial component $(\mathrm{T})$, with asymmetric narrowing of the medial femorotibial joint space suggestive of underlying polyethylene liner wear (black arrows).
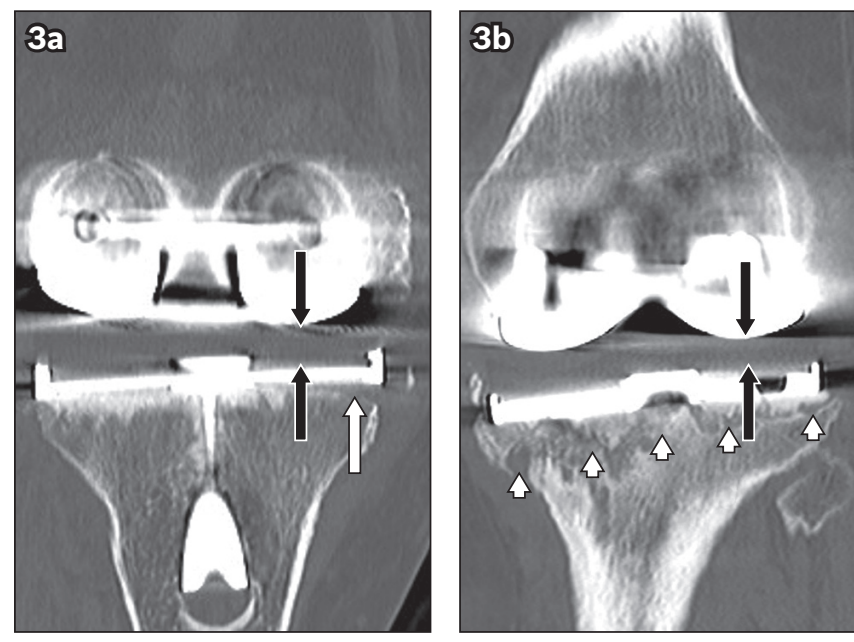

Fig. 3 (a) Coronal CT image of the left knee in a 72-year-old woman with post-total knee replacement aseptic loosening shows a focal periprosthetic lucency (white arrow) underlying the lateral base plate of the tibial component, which is in keeping with early loosening, and mild asymmetric narrowing of the lateral femorotibial joint space suggestive of early polyethylene liner wear (black arrows). (b) Follow-up CT image taken 18 months later shows interval progression of prosthesis loosening and liner wear, as seen by increased periprosthetic lucency (arrowheads) and further reduction in the lateral femorotibial joint space (black arrows).

poor bone stock or varying combinations of these factors. ${ }^{(7,8)}$ Radiographic and CT findings of loosening include wide (>2 mm) or progressively enlarging periprosthetic lucency, component migration and subsidence (Figs. 2 \& 3). This is distinct from stress shielding, which is a loss of bone mineralisation following reduction in mechanical loading of the periprosthetic bone. Unlike aseptic loosening, stress shielding is a non-progressive, radiographically stable, periprosthetic lucency; it is most commonly seen underlying the anterior and posterior flanges of the femoral component or subtending the tibial tray. ${ }^{(7)}$ Implant infection and aseptic loosening cannot be reliably distinguished 

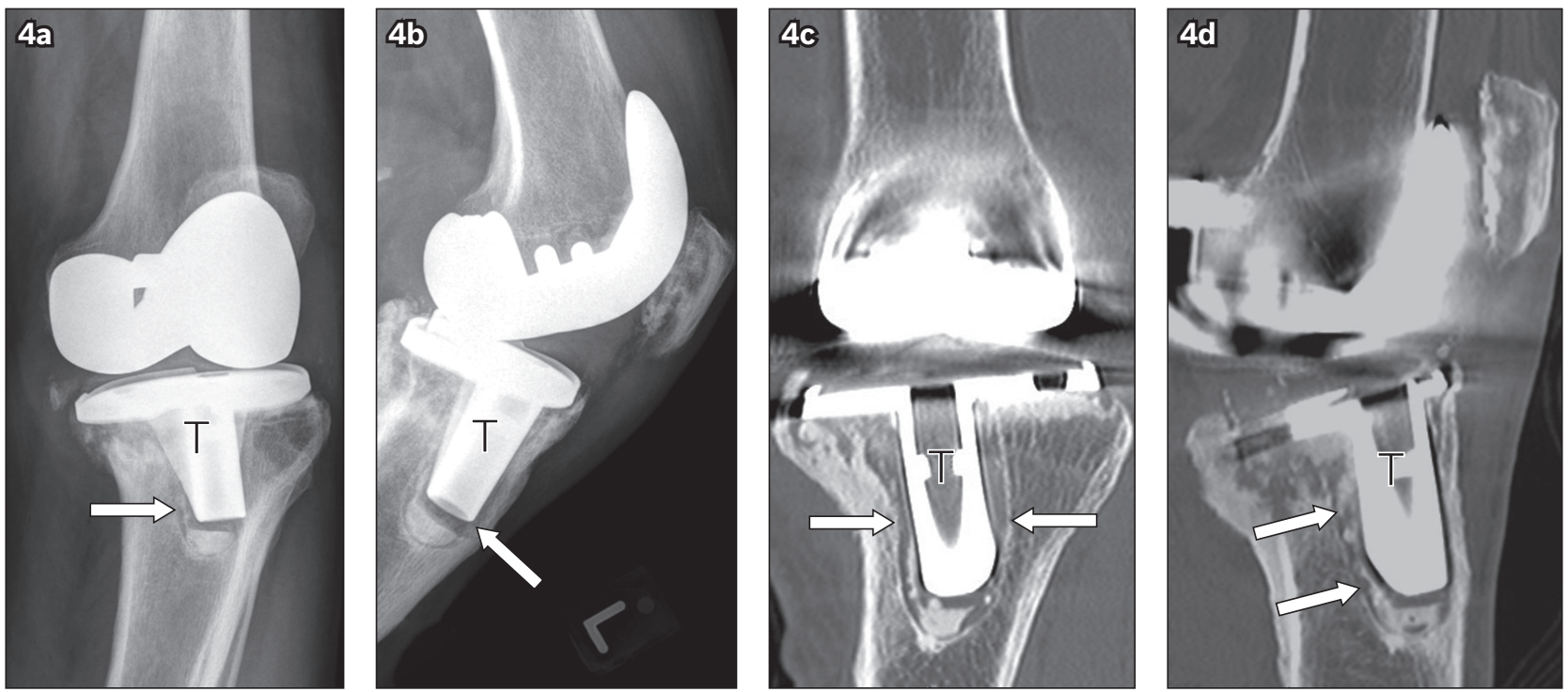

Fig. 4 (a) Anteroposterior and (b) lateral radiographs and (c) coronal and (d) sagittal CT images of the left knee in a 69-year-old man with aseptic loosening after total knee replacement show significant periprosthetic lucency (arrows) with respect to the tibial component ( $\mathrm{T}$ ), posteromedial subsidence, and resultant varus angulation of the knee joint.
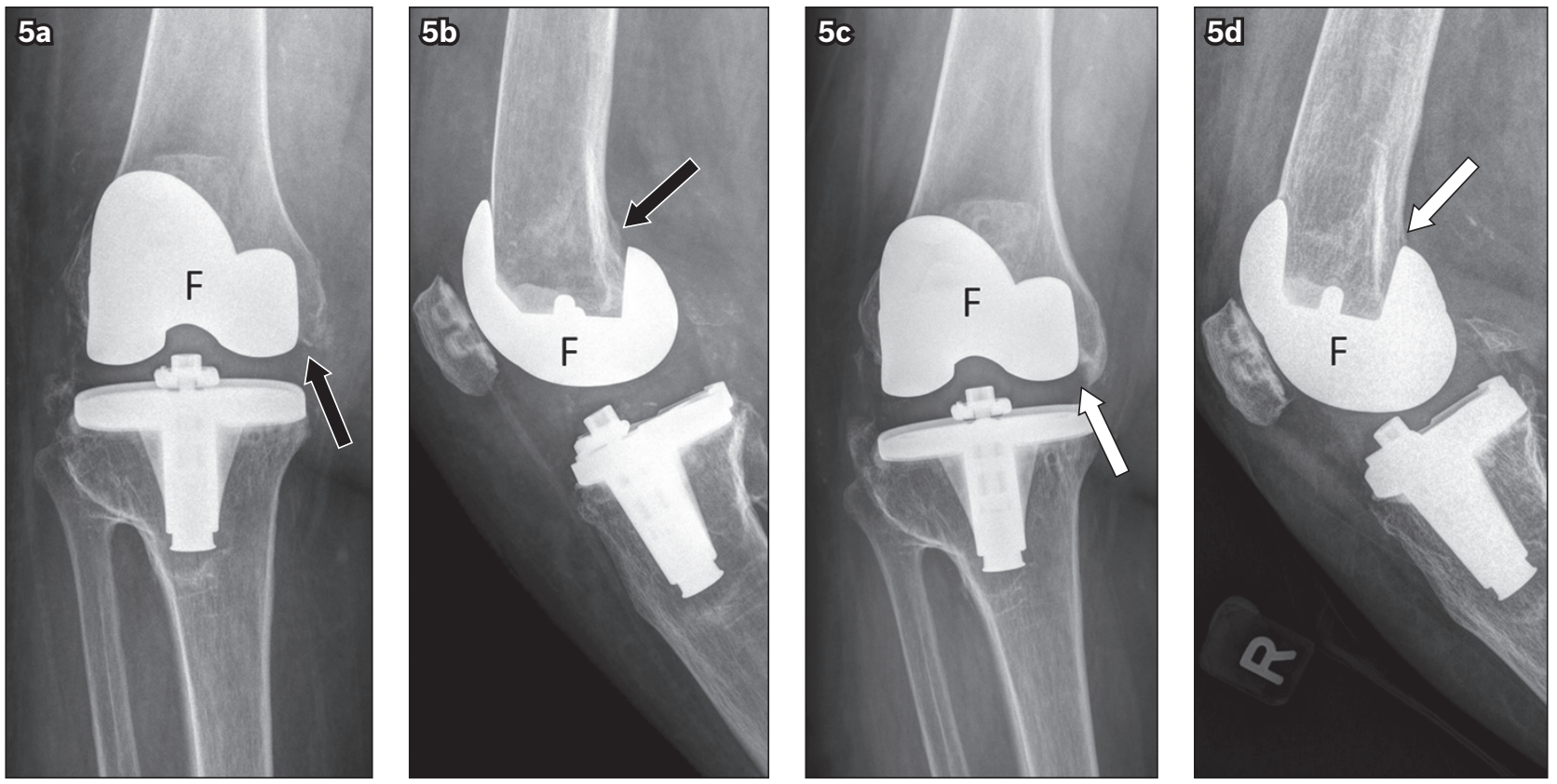

Fig. 5 (a) Anteroposterior and (b) lateral radiographs show the right knee of a 68-year-old woman in the immediate postoperative period. (c) Anteroposterior and (d) lateral follow-up radiographs of the right knee five years later show subsidence of the femoral component (white arrows) when compared to the prior radiographs (arrows in a \& b), with resultant varus alignment of the knee.

from each other on radiography, and hence infection should always be considered when loosening is present. ${ }^{(7,8)}$

Subsidence following TKR usually refers to 'sinking' or 'settling' of the tibial tray into the tibial plateau due to loss of bone substance beneath the tibial tray. This is more commonly observed in the medial tibial plateau, with subsequent varus malalignment of the knee joint (Fig. 4). Less commonly, femoral subsidence may occur with shifting of the femoral component (Fig. 5). ${ }^{(7)}$

Polyethylene liner wear is a frequent late complication of TKR, especially in patients with prostheses that have metal backing and non-congruent articular surfaces, although there have been improvements in polyethylene liner durability over the years. ${ }^{(7,8)}$ Moderate to severe wear appears as obvious joint space narrowing, with progressive worsening varus or valgus deformity on knee radiographs during follow-up (Figs. 2 \& 3). However, mild subclinical wear is often subtle and appreciated only when weight-bearing anteroposterior and lateral radiographs of the affected knee are compared with previous images. ${ }^{(7)}$

Osteolysis, or 'particle disease', is a cytokine-mediated inflammatory reaction that can occur secondary to particles arising from polyethylene, cement or metal. This usually begins as a proliferative synovitis and progresses into a geographic osteolysis, which causes loosening of the implants. ${ }^{(6-8)}$ Non-contrast CT may be considered for imaging evaluation when osteolysis is 
suspected, as it has greater sensitivity than radiography, allowing for accurate quantification of disease extent (Fig. 6). MR imaging with metal artefact-reduction techniques may also be useful in demonstrating proliferative synovitis associated with osteolysis before significant bone loss occurs. ${ }^{(6,7)}$

Instability and dislocation may also be present. Instability can be defined as abnormal displacement of a knee prosthesis that leads to clinical failure necessitating early revision. This may be secondary to various factors, including improper prosthesis selection, ligament tears, imbalance of the stabilising soft tissue structures at surgery, polyethylene wear and implant migration. ${ }^{(6-8)}$ Dislocation is the most serious manifestation (Fig. 7), but its prevalence has decreased to $0.15 \%-0.50 \%$ due to newer implant technology. ${ }^{(7)}$ Varus-valgus stress radiography can help to indicate the extent of ligamentous laxity, on top of assessing the alignment of the TKR components.

Prosthetic joint infection is a potentially devastating complication following TKR, and expedient diagnosis remains key in preserving joint function, limiting associated tissue damage and avoiding systemic sepsis. ${ }^{(6)}$ Clinical symptoms and signs such as wound drainage, erythema, joint swelling and fever may prompt the clinician to suspect underlying infection. Findings on radiographs may range from normal to overt bony destruction and may mimic osteolysis from particle disease or aseptic loosening (Fig. 8). The radiographic finding of gas within the joint or surrounding soft tissue is specific but rare. ${ }^{(7)}$ Increased uptake in all three phases of a 99-mTc triple-phase bone scan is characteristic of an infected TKR. ${ }^{(7,8)}$ On MR imaging, the presence of lamellated T2-weighted hyperintense infective synovitis may be used to differentiate infection from the frond-like hypertrophied synovium seen in particle disease. ${ }^{(8,9)}$

Periprosthetic fractures related to TKR (Fig. 9) may occur intraoperatively or postoperatively. There are multiple
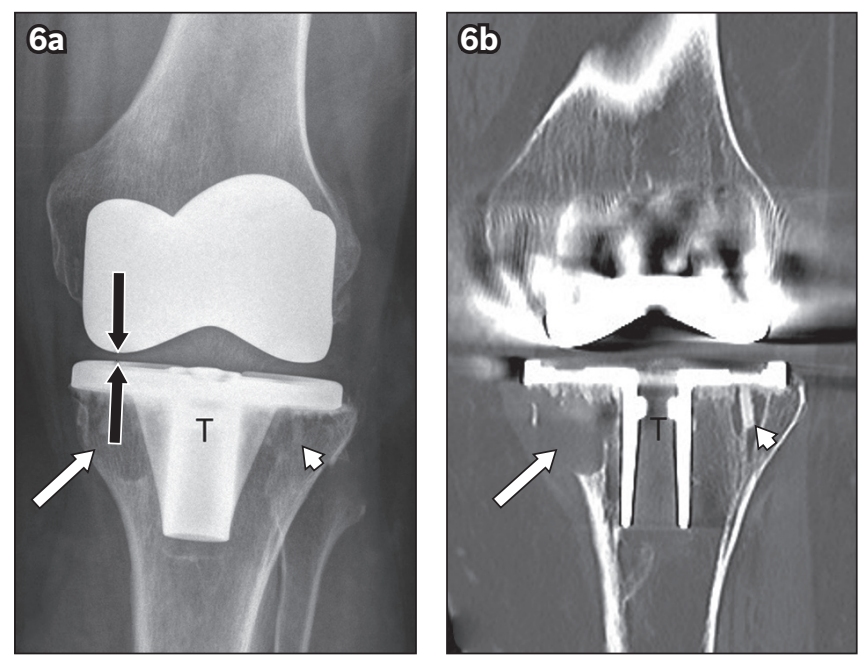

Fig. 6 (a) Anteroposterior radiograph of the left knee in a 61-year-old woman with post-total knee replacement particle disease shows geographic lucent areas of osteolysis underneath the medial base plate (white arrow) of the tibial component $(T)$ and, to a lesser extent, the lateral base plate (arrowhead), with narrowing of the medial femorotibial compartment (black arrows), in keeping with polyethylene liner wear. (b) Coronal CT image shows cortical erosion associated with the osteolysis subtending the medial base plate (arrow) of the tibial component, and CT more clearly delineates the osteolysis surrounding the fixation screw used to secure the lateral base plate (arrowhead). Suparapatellar joint synovitis was also evident on CT (not shown).
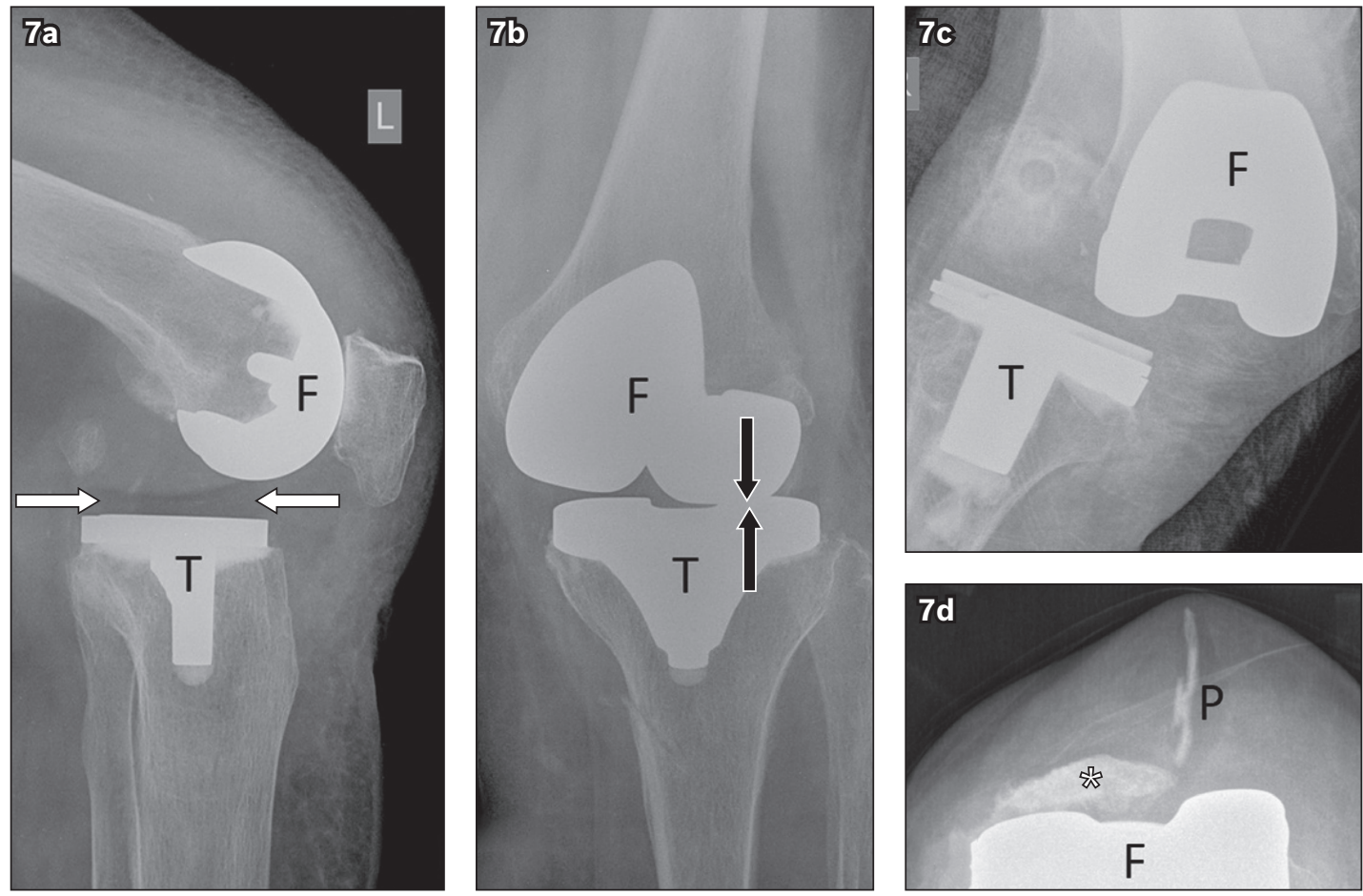

Fig. 7 (a) Lateral radiograph of the left knee in an 82-year-old woman with post-total knee replacement (TKR) instability shows anterior subluxation of the femoral component (F) relative to the polyethylene liner (arrows) and tibial component ( $T$ ) due to rupture of the posterior cruciate ligament in a cruciate-retaining TKR with a loose flexion gap. (b) Anteroposterior radiograph of the left knee in the same patient shows narrowing of the lateral femorotibial compartment (arrows) in keeping with polyethylene liner wear. (c) Anteroposterior radiograph of the right knee in a 78-year-old woman with post-TKR instability shows medial dislocation of the femoral component relative to the tibial component. (d) Skyline radiograph of the right knee in an 86-year-old woman shows detachment and dislocation of the patellar component (P) from the native patella (asterisk). 

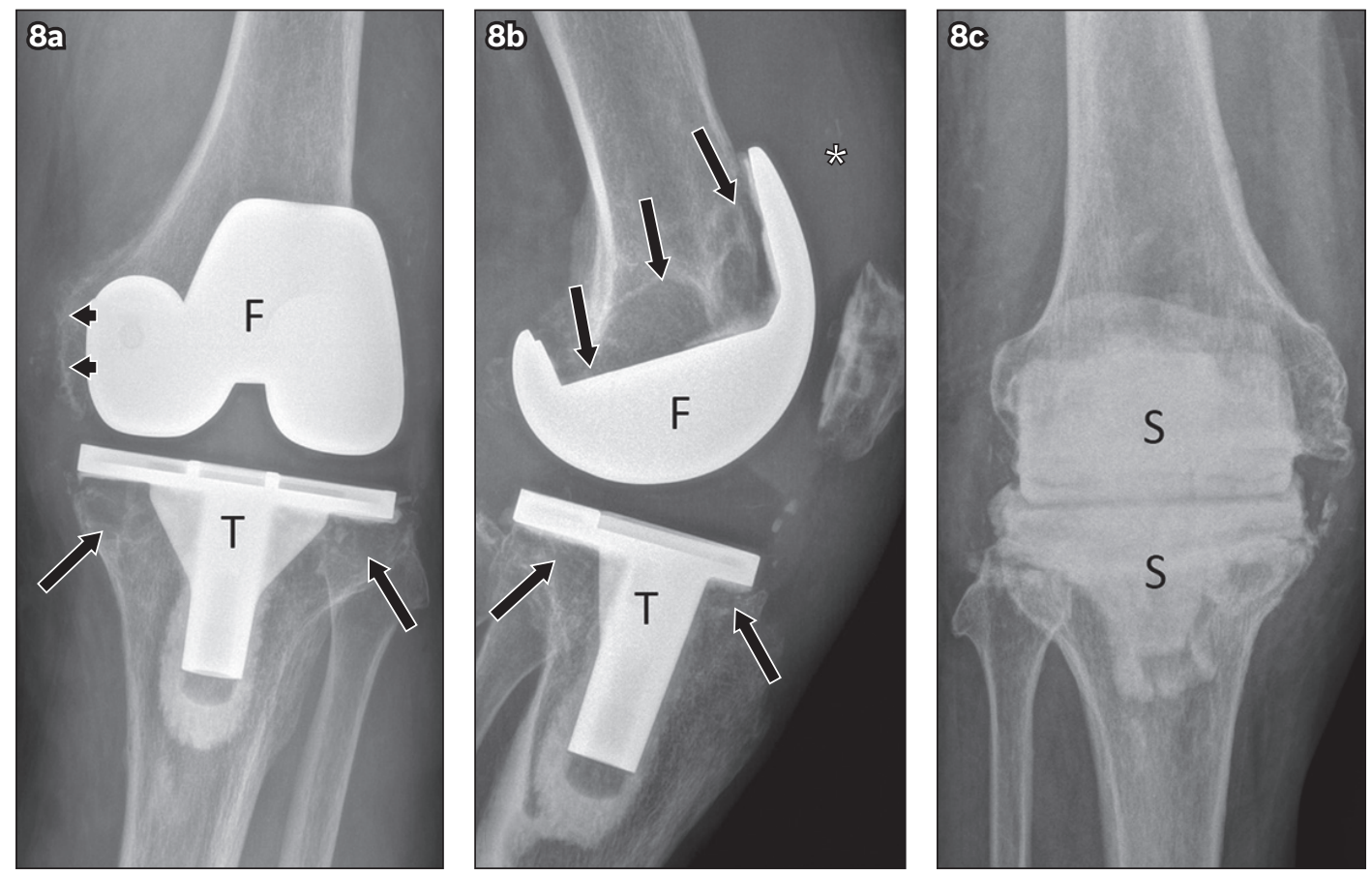

Fig. 8 (a) Anteroposterior and (b) lateral radiographs of the left knee in a 78-year-old man with prosthetic joint infection show areas of osteolysis along the implant bone and cement bone interfaces of the femoral (F) and tibial (T) components (arrows); periosteal reaction along the medial femoral condyle (arrowheads); and a suprapatellar joint effusion (asterisk). (c) Anteroposterior radiograph of the right knee in a 76-year-old man with post-total knee replacement infection shows interim insertion of antibiotic cement spacers (S) following removal of the femoral and tibial components before performing definitive revision arthroplasty.
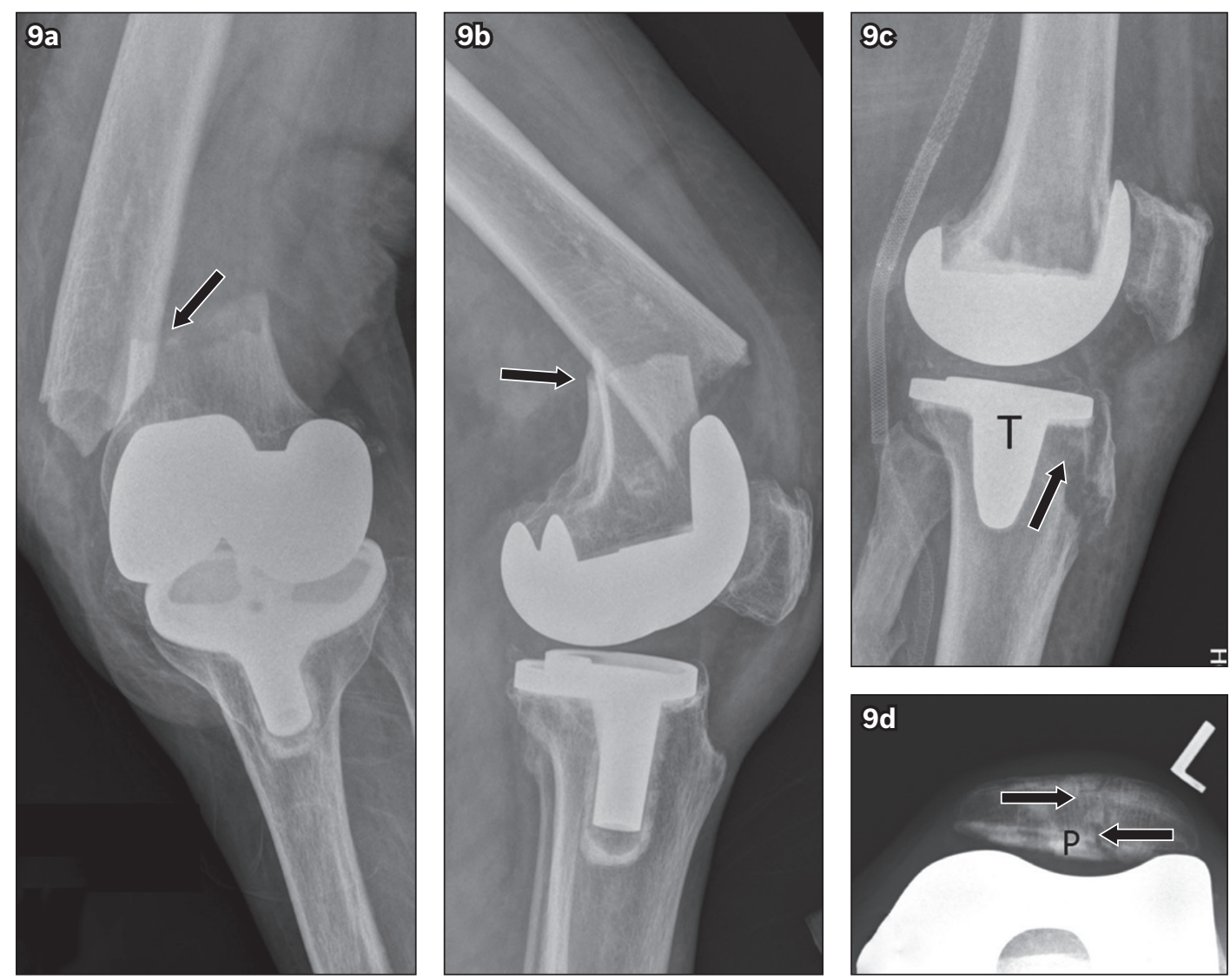

Fig. 9 (a) Anteroposterior and (b) lateral radiographs of the left knee in a 71-year-old woman with previous total knee replacement (TKR) show an acute, displaced periprosthetic supracondylar femoral fracture (arrows). (c) Lateral radiograph of the left knee in a 75-year-old woman with prior TKR shows an acute, mildly displaced periprosthetic proximal tibial fracture (arrow) which extends to the anterior proximal stem of the tibial component ( $\mathrm{T}$ ). (d) Skyline radiograph of the left knee in a 73-year-old woman shows an acute, undisplaced periprosthetic fracture (arrows) of the patella that extends to the patellar component $(P)$. TKR: total knee replacement. 
predisposing factors for periprosthetic fractures, including the presence of poor bone stock, osteopenia, osteolysis and osteonecrosis. ${ }^{(6,8)}$ The most frequently encountered fracture (incidence $0.3 \%-2.5 \%$ ) occurring in juxtaposition to a TKR is a supracondylar fracture of the femur. Periprosthetic tibial fractures and periprosthetic patellar fractures are uncommon.

In conclusion, given the increasing number of knee arthroplasties performed and the decreasing patient age at implantation, there is escalating demand for an accurate diagnosis to be made and root causes identified for dysfunctional arthroplasties. Together with clinical assessment, imaging guides the treatment of symptomatic or dysfunctional joints following TKR, as well as monitoring of at-risk patients. Therefore, it is imperative that the attending orthopaedic surgeon and reporting radiologist are cognisant of the aforementioned potential complications of TKR.

\section{REFERENCES}

1. Hozack WJ, Rothman RH, Booth RE Jr, Balderston RA. The patellar clunk syndrome. A complication of posterior stabilized total knee arthroplasty. Clin Orthop Relat Res 1989; 241:203-8.

2. Koh YG, Kim SJ, Chun YM, Kim YC, Park YS. Arthroscopic treatment of patellofemoral soft tissue impingement after posterior stabilized total knee arthroplasty. Knee 2008; 15:36-9.

3. Marchand AJ, Proisy M, Ropars M, et al. Snapping knee: imaging findings with an emphasis on dynamic sonography. AJR Am J Roentgenol 2012; 199:142-50.

4. Heyse TJ, Chong LR, Davis J, et al. MRI diagnosis of patellar clunk syndrome following total knee arthroplasty. HSS J 2012; 8:92-5.

5. Yau WP, Wong JWK, Chiu KY, Ng TP, Tang WM. Patellar clunk syndrome after posterior stabilized total knee arthroplasty. J Arthroplasty 2003; 18:1023-8.

6. Fritz J, Lurie B, Potter HG. MR imaging of knee arthroplasty. Radiographics 2015; 35:1483-501.

7. Mulcahy H, Chew FS. Current concepts in knee replacement: complications. AJR Am J Roentgenol 2014; 202:W76-86.

8. Mar WA, Tan I, Song A, et al. Update on imaging of knee arthroplasties: normal findings and hardware complications. Semin Musculoskelet Radiol 2019; 23:e20-e35.

9. $\mathrm{Li} \mathrm{AE}$, Sneag DB, Greditzer HG 4th, et al. Total knee arthroplasty: diagnostic accuracy of patterns of synovitis at MR imaging. Radiology 2016; 281:499-506. 


\section{SINGAPORE MEDICAL COUNCIL CATEGORY 3B CME PROGRAMME} (Code SMJ 202009B)

Question 1. Regarding patellar clunk syndrome:

(a) It is the most common cause of perceivable 'snapping' of the knee following total knee replacement (TKR).

(b) It is due to impingement of suprapatellar nodular scar tissue at the prosthetic intercondylar notch with knee flexion, which displaces with knee extension.

(c) Patients often complain of symptoms during knee extension at about $80^{\circ}-90^{\circ}$.

(d) Low-lying patella (patella baja) is a risk factor.

Question 2. Which of the following applies to imaging assessment of patellar clunk syndrome?

(a) Magnetic resonance (MR) imaging is the preferred imaging modality of choice.

(b) On ultrasonography, a fibrous nodule can be seen to abruptly displace from the intercondylar notch into the suprapatellar region with knee extension.

(c) Computed tomography (CT) is a useful modality of assessment.

(d) MR imaging assessment may be limited by artefacts from the metallic prosthesis.

Question 3. Regarding aseptic loosening of joint prosthesis:

(a) Poor bone stock is a potential contributing factor.

(b) It cannot be reliably distinguished from implant infection on radiography.

(c) Imaging findings include progressively enlarging periprosthetic lucency.

(d) Subsidence of the femoral component is more common than that of the tibial component after total knee replacement.

Question 4. Regarding prosthetic joint infection:

(a) Initial radiographs may be normal.

(b) Radiographic findings in an infected joint may mimic aseptic loosening or osteolysis from particle disease.

(c) Presence of gas within the joint or surrounding soft tissue on radiographs is a common feature.

(d) A 99-mTc triple-phase bone scan is helpful to detect an infected TKR.

Question 5. Regarding other complications in TKR:

(a) Polyethylene wear is a frequently encountered early complication of TKR replacement.

(b) Mild subclinical polyethylene wear is often subtle and best appreciated when radiographs are compared with prior images.

(c) Polyethylene, cement or metallic particles can result in an inflammatory reaction and osteolysis.

(d) Non-contrast CT may be considered when osteolysis is suspected.

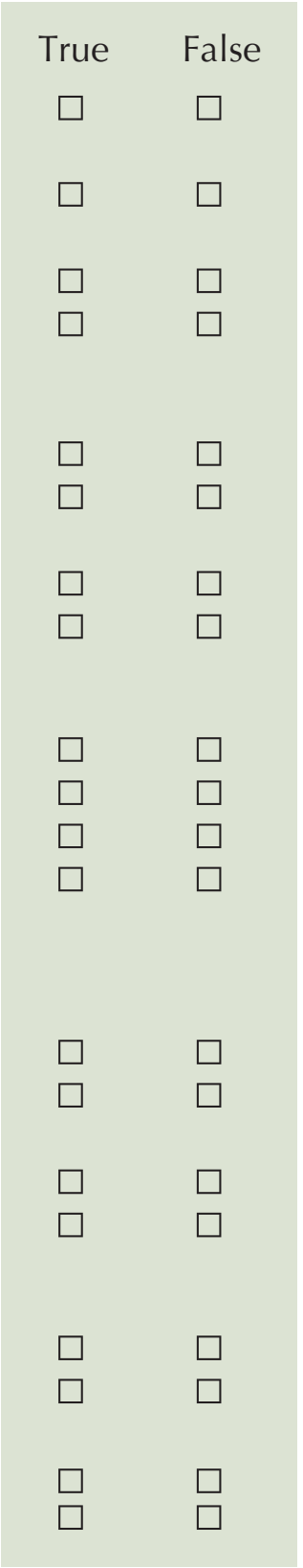

\section{Doctor's particulars:}

Name in full:

MCR no.:

Specialty:

Email:

\section{SUBMISSION INSTRUCTIONS:}

Visit the SMJ website: http://www.smj.org.sg/current-issue and select the appropriate quiz. You will be redirected to the SMA login page.

For SMA member: (1) Log in with your username and password (if you do not know your password, please click on 'Forgot your password?'). (2) Select your answers for each quiz and click 'Submit'.

For non-SMA member: (1) Create an SMJ CME account, or log in with your SMJ CME username and password (for returning users). (2) Make payment of SGD 21.40 (inclusive of 7\% GST) via PayPal to access this month's quizzes. (3) Select your answers for each quiz and click 'Submit'.

RESULTS:

(1) Answers will be published online in the SMJ November 2020 issue. (2) The MCR numbers of successful candidates will be posted online at the SMJ website by 10 November 2020. (3) Passing mark is $60 \%$. No mark will be deducted for incorrect answers. (4) The SMJ editorial office will submit the list of successful candidates to the Singapore Medica Council. (5) One CME point is awarded for successful candidates. (6) SMC credits CME points according to the month of publication of the CME article (i.e. points awarded for a quiz published in the September 2020 issue will be credited for the month of September 2020, even if the deadline is in November 2020).

Deadline for submission (September 2020 SMJ 3B CME programme): 12 noon, 3 November 2020. 